\title{
Review on An Article of Life Lying in Sport is Misleading from Critical Thinking Perspective Hao-wen FENG ${ }^{1, a}$ and Wei-min OUYANG ${ }^{2, b,{ }^{*}}$ \\ ${ }^{1,2}$ Shanghai University of Political Science and Law, Shanghai, China \\ afenghaowen@shupl.edu.cn, boywm@shupl.edu.cn
}

Keywords: Critical thinking, Anecdotal evidence, Hasty generalization, False premise.

\begin{abstract}
On March 25, 2010, Dr Zheng Weijing published an article in his web, which is called that life lying in sport is misleading. This article sparked a lot of controversy in cyber space, which was forwarded and commented wildly in circle of friends and webs. However, most of the discussions were emotional more than rational. In this paper, we made comments on this article from the perspective of critical thinking.
\end{abstract}

\section{Introduction}

On March 25, 2010, Dr Zheng Weijing published an article in his web, which is called that life lying in sport is misleading. This article sparked a lot of controversy in cyber space, which is forwarded and commented wildly in circle of friends and webs. Dr. Cheng believes that the reason why people can not live long life is due to the wrong ideas of longevity, and points out his four ideas, such that life exists in sport is misleading, to eat much for breakfast Will shorten the life span, too much sexual life will shorten the life span, too much hope will shorten life span. This article sparked a lot of controversy in cyber space, which is forwarded and commented wildly in circle of friends and weibos. However, most of the discussions are emotional more than rational. In this paper, we will make comments on this article from the perspective of critical thinking.

\section{Thinking Fallacy of Anecdotal Evidence}

Anecdotal evidence or hearsay evidence refers to evidence from hearsay and story. Some hearsay often have rich details, vivid and impressive; some cases in the form of news and gossip were passed again and again, let people who was infused for long time will believe that it is true.

Dr Zheng Weijian said as follows:

Paying a little attention, it is not hard to know, among the early death in recent years, many people died of movement in recent years. Many people fear of illness, hope to have a good body, so, they exercise all day. Ma Hua, a beautiful young fashion female coach appeared in a CCTV program "5 minutes for bodybuilding", such a woman, who every day advocated exercise every day and good health, died of illness so early.

Although the story is very vivid, but, to set an example is not to make an argument. Vivid cases can be infected with our emotions, and distract our attention, and let us forget to look for other relevant evidence. The attempt to use individual stories to replace logic argument or strong evidence is a logical fallacy, which is called anecdotal evidence from the critical thinking perspective.

Dr. Zheng is trying to use the case of Ms. Ma hua to prove his viewpoint that life 
exists in sport is misleading, and thus committed a thinking fallacy called as anecdotal evidence.

Furthermore, Dr Zheng put forward that rest is the fundamental to prolong life and living as follows:

Among a famous longevity village, people over the age of 100 are everywhere around this village, the oldest old people is 120 years old. Looking at these elders, we can find that none of these longevity old people has a long life because of sports, instead, they live in the rest from the young time, and rest is the fundamental to prolong life and living!

Here, Dr. Cheng once again use anecdotal evidence to prove his viewpoint, therefore, he once again committed the fallacy of thinking called as anecdotal evidence. Vivid case is easy to cause emotional resonance, but it's just moving anecdotes rather than evidence. Setting example does not take the place of argument.

\section{Thinking Fallacy of Hasty Generalization}

A hasty generalization is a fallacy in which a conclusion is not logically proved by sufficient or unbiased evidence. Simply put, a hasty generalization is a broad claim based on too-limited evidence. Hence, hasty generalization is also called insufficient sample, converse accident, faulty generalization, biased generalization.

By definition, an argument based on a hasty generalization always proceeds from the particular to the general. Dr. Zheng does hump to a general conclusion, i.e., too much and too high ideal will lead to reduce life span, from some individual instances. He said as follows:

Early on July 22, 2008, Zhang Shengyu, chairman of Beijing TongRenTang co., LTD., suddenly died of a heart attack, who was only 39 years old. TongRenTang co., known as the "medicine in mao-tai", lost his younger leader. Zhang Shengyu has always been in bad health, and usually work hard, since April 2007 acts as chairman of the company until now, just more than a year, he has moved to death, and let people sadly sigh.

In this passage, Dr. Cheng proved his viewpoint, i.e., too much and too high ideal will lead to reduce life span, by the sudden death of Zhang Shengyu. This argument committed a thinking fallacy of hasty generalization. Although there are many reasons, such as heart disease and hard work and so on, for his early death, Dr. Zhang attributed sudden death of heart attack of Zhang Shengyu to his too much and too high ideal. This kind of thinking method, which only identified one factor from a number of factors to be the cause of the event, is called as a thinking fallacy of complex cause. Therefore, Dr. Cheng also committed a thinking fallacy of complex cause in the above passage.

Dr Zheng Weijian applied the same wrong argument again. He said as follows:

This situation has become a common phenomenon in today's society. Here, Dr. Zheng might as well cite some untimely death in recent years:

In July 2001, Tsingtao Brewery Co., LTD., vice chairman and general manager Mr. Peng Zuoyi died unfortunately, aged 56.

In April 2004, Ericsson China President Yang Mai died suddenly, at the age of 54.

In March 2004, Beijing DaZhong electric appliance Co., LTD., general manager $\mathrm{Hu} \mathrm{Kai}$, died of a heart attack at the age of 52.

In February 2006, GanYue expressway Co., LTD., chairman of Wu Shaoming died at the age of 56.

On June 5, 2009, the famous news anchorman of CCTV "News Broadcast", Luojing 
die of an illness in Beijing. He was 48.

Obviously, Dr. Cheng tried to prove his viewpoint, i.e., too much and too high ideal will lead to reduce life span, by citing the untimely death of these successful people. However, this kind of argument drawn from the particular instance to a general conclusion is cursory, is of no logical necessity, which committed a thinking fallacy of hasty generalization. Moreover, the main cause of death for these successful people in fact, is a variety of diseases, not busy word. So, Dr. Cheng intentionally omitted the real main reason, and committed a thinking fallacy of insignificant cause.

\section{Thinking Fallacy of False Premise}

A false premise is a wrong proposition that constitutes the basis of a hypothetical proposition. Since the premise is wrong, the hypothetical proposition is also wrong, therefore, the conclusion drawn by the hypothetical proposition is incorrect. Although this argument is logically valid, but the conclusion is wrong, because its first premise is false.

Dr Zheng Weijian said as follows:

Humans and animals are the same, the number of heart beats, the number of gastrointestinal peristalsis, the number of breaths and the number of endocrine reactions was all fixed. Pay attention to this "fixed number". In other words, the beating of the heart, gastrointestinal motility, breath and endocrine responses are relatively fixed number, to eat much for breakfast is bound to increase the number in per unit time, as a result, the relative life span is reduced.

Here, Dr. Zheng first asserted the number of heartbeats for human life is a constant, and took this assertion as a premise, then draw a hypothetical proposition by this premise, i.e., if any behavior causes the number of heartbeats of someone in unit time heartbeat to be increased, then the behavior will lead to the life span of the person is relatively shorten, and to eat much for breakfast will accelerate the heartbeat frequency. So, to eat much for breakfast will cause life span of the person to be relative reduced.

Since there is no scientific basis for the assertion that the number of heartbeats in a person's life is a fixed number, the assertion is rested on a basis of his conjecture. The conclusion drawn by this conjecture, to eat much for breakfast will shorten the life span, is incredible, and thus committed a thinking fallacy called as false premise.

Dr Zheng Weijian employed the same wrong argument again. He said as follows:

Sexual life is also a violent movement, compared to the treadmill, morning run, mountain climbing, playing sports and other sports, the sport amount of sexual life is more violent, more exciting. However, here is the sentence that Dr. Zheng said earlier, from the analysis with numerology and Li-theory, everything is a fixed number. How many times have you lived your life, how many times you should have in your life, how many the opposite sex in your life, and so on, each of these data is a constant number. If you increase the number in per unit time, then, obviously, your life will be shortened.

He still employed the assertion that the number of heartbeats for human life is a constant as the premise for hypothetical reasoning, and the assertion has no scientific basis. By making use of incorrect premise, the conclusion drawn is not incredible, even if the reasoning process is correct. Here, Dr. Zheng committed a thinking fallacy as before.

In this passage, Dr. Zheng invoked the so-called "numerology" and "Li-theory" which do not have any scientific basis, therefore, he also committed a thinking fallacy 
of appealing to authority.

\section{Conclusions}

In conclusion, this article on the "life lying in sport is misleading", published by Dr. Zheng Weijian in his micro-blog, has a lot of thinking fallacies. The existence of these thinking fallacies is enough to completely eliminate credibility of the article. From the type of thinking fallacy perspective, Dr. Zheng had mainly committed anecdotal evidence, hasty generalization and false premise; from the expression method of misleading the readers, Dr. Zheng had tried to utilize vivid examples and false premise to mislead the readers. These methods of argument not only are absurd on the logical, but also are unethical on the thinking trait, which are totally unacceptable.

\section{References}

[1] Zheng Weijian, Life exists in sport is misleading, March 25, 2010. http://blog.sina.com.cn/s/blog_50350d150100h4k5.html.

[2] M.Neil Browne,Stuart M.Keeley, translated by Zhao yufang, Asking The Right Questions: A Guide to Critical Thinking(10th Edition), China Machine Press, Published in December 2012.

[3] Richard Paul, Linda elder, translated by Hou yubo, Critical Thinking Tools for taking change for your learning and your life, 3rd Edition, China Machine Press, Published in December 2012. 